\title{
Langzeitbeobachtungen und Betriebserfahrungen mit einem 40 Jahre alten Speicherbecken der Kraftwerksgruppe Fragant
}

\begin{abstract}
Zusammenfassung: Um die Sicherheitsbeurteilung und das Verständnis für das Verformungsverhalten der Damm- und Einschnittsböschungen $\mathrm{zu}$ verbessern, werden verschiedene Überwachungsmethoden eingesetzt, die im Aufsatz detailliert ausgeführt sind. Das Speicherbecken Haselstein bildet seit 40 Jahren ein Sammelbecken bzw. einen Tagesspeicher für zwei Bachbeileitungen mit einem Speichervolumen von $40000 \mathrm{~m}^{3}$ und einer maximalen Tiefe von $18 \mathrm{~m}$. Da das Becken sehr nah zum Berghang auf einem Felsplateau errichtet wurde, zeigten sich bald nach der Inbetriebnahme Verformungen der Asphaltoberflächendichtung an der bergseitigen Böschung. Neben der Verdichtung von geodätischen Messpunkten, Untergrunderkundungen etc. begann eine Firma im Auftrag der Kelag 2004 die Asphaltoberfläche mittels einer Spezialkamera zu dokumentieren und die 3D-Bilder mit einer speziellen Software auszuwerten. Messungen in zeitlichen $\mathrm{Ab}$ ständen sollen Veränderungen von Materialeigenschaften und sonstige abnormale Veränderungen frühzeitig aufspüren, die zunehmende Sickerwassermengen infolge Dichtungsschäden verbunden mit inneren Erosionen, nicht mehr substabile Hangkriechbewegungen oder andere Versagensszenarien auslösen könnten.
\end{abstract}

Experience gathered in the long-term observation of a 40-year old reservoir of the Fragant Hydro Scheme

Summary: The paper describes the methodology, operative techniques and other aspects that are going to be used for dam safety assessment procedures. It goes without saying that it is necessary to monitor the behaviour of the structures. The Haselstein reservoir is a component of KELAG's Fragant Group of hydropower facilities which has been in operation for more than 40 years. Since 1979 ongoing deformations in the asphaltic concrete lining have been observed. In 2004 KELAG started a documentation compaign of the asphalt concrete lining using a special high resolution 3D imaging system and according 3D assessment software. Different measurements

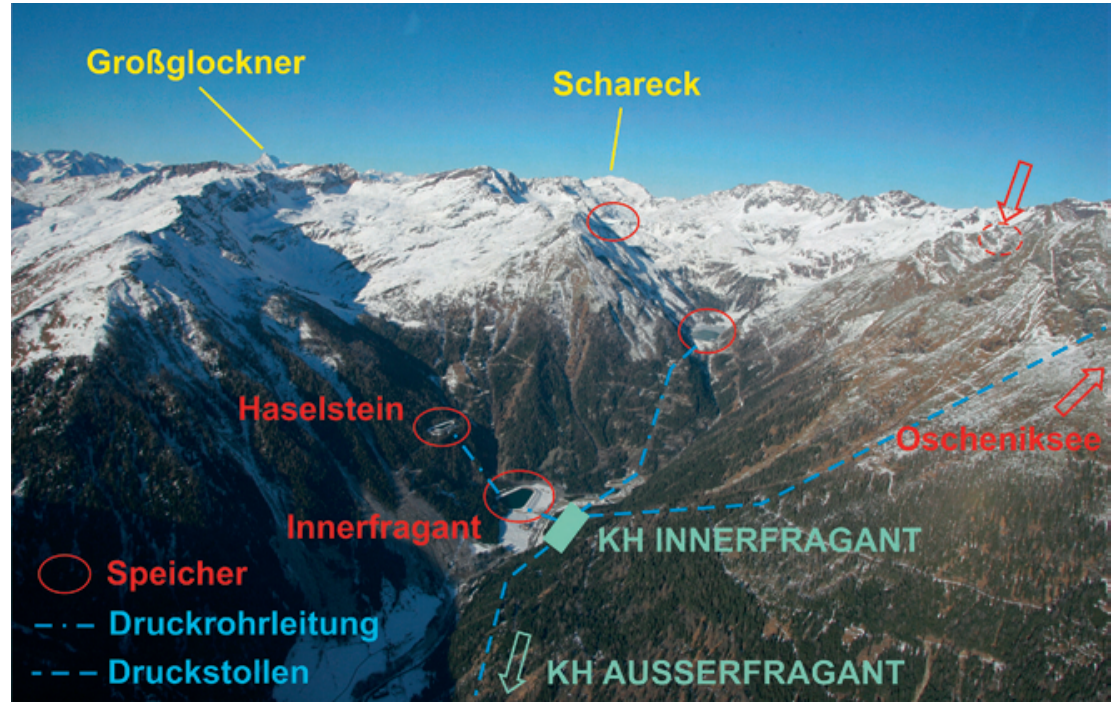

Abb. 1: Kraftwerksanlage Fragant Bereich Innerfragant.

should detect changes of material properties as well as anomalous variations, which may relate to increasing seepage, internal erosion, increasing deformation of slopes or defects in foundation. However, applying several automatic surveillance gauges and methods on the Haselstein are challenging to carry out the dam safety assessment to minimize failure risk.

\section{Einleitung}

Die KELAG betreibt im Nordosten von Kärnten in den Hohen Tauern mehrere Kraftwerke mit Speicheranlagen, die vor mehr als 30 Jahren erbaut wurden und stellt in 4 Krafthäusern über 330 MW Leistung bereit. Das Speicherbecken Haselstein dient als Ausgleichsspeicher für zwei Bachbeileitungen aus einem $21,5 \mathrm{~km}^{2}$ großen Einzugsgebiet. $250 \mathrm{~m}$ über dem Krafthaus Innerfragant im Talabschluss auf einem Felsplateau gelegen, optimiert das Becken als Vordruckspeicher die Wasserverlagerung in den auf 2300 müA gelegenen Hauptspeicher Oschenik.

Der Bau des Kunstspeichers auf der Haselsteinalm hatte auf die geologischen Verhältnisse des Felsuntergrundes besondere Rücksicht zu nehmen. Der Dammfuß wurde von der Böschungskante maximal zum Hang verschoben, um die äußerste Felskante nicht zusätzlich zu belasten. Die Schüttung des Außendammes erfolgte aus dem Materialausgleich des Abtrages von der Bergseite. Das Becken ist dem damaligen Stand der Asphalttechnik entsprechend mit einem zweilagigen Dichtungselement, $8 \mathrm{~cm}$ Asphaltmischgut auf entsprechendem Filter- und Binderuntergrund gebettet, ausgekleidet worden.

Einige Jahre nach der Inbetriebnahme hat sich eine wulstartige Verformung an der bergseitigen Asphaltoberfläche gezeigt, die 1979 örtlich saniert wurde. Als Grund für die Bildung des Wulstes wurde das Versteilen der bergseitigen Hangböschung beim Bau des Beckens angenommen. Aus den zwischen 1983 und 2008 regelmäßig durchgeführten geodätischen Messungen lassen sich jährliche Verformungsbeträge zwischen 25 und $40 \mathrm{~cm}$ feststellen. Der bergseitige Beckenrand sowie Teile der Asphaltböschung erleiden eine jährliche Verschiebung von bis zu $16 \mathrm{~mm}$ in Richtung Speicherbecken.

Um die flächenhafte Verschiebung der Asphaltoberfläche zu erfassen, wurde vor mehr als 4 Jahren mit einer hochauflösenden Linienkamera die Asphaltoberfläche dokumentiert. Die digitalen Daten können mittels spezieller Software und 
kombiniert mit den geodätischen Messergebnissen von ausgesuchten Oberflächenpunkten in 3D-Ansichten dargestellt werden und von verschiedensten Perspektiven auf dem Bildschirm analysiert werden. Die Aufnahmen dokumentieren auch die Alterung der Asphaltoberfläche und dienen bei einem Sickerwasseranstieg der schnelleren Leckortung in der Asphaltoberfläche. Die Sickerwässer werden über den Drainagefilter gesammelt und über ein bekriechbares Rohr von der Beckenmitte bis zu einem Messwehr mit automatischer Übertragung und separaten Grenzwertschaltern ausgeleitet. Bei einer Grenzwertüberschreitung ist vom Personal der Hauptschaltleitung in Klagenfurt der Talsperrenverantwortliche zu verständigen. Ihm obliegt die sicherheitsrelevante Bewertung sowie Veranlassung der weiteren Maßnahmen. Das verfeinerte Messprogramm dient der besseren Erfassung von Parameterveränderungen im Gefahrenfall und schafft zeitliche Reserven für die Aufrechterhaltung einer störungsfreien Funktion der Anlage.

Die Grenzwerte sind so ausgewählt, dass eine Auslösung noch weit entfernt von einem katastrophenähnlichen Versagen einer Sperre ist. Die Verständigung soll den Talsperrenverantwortlichen rechtzeitig auf eine Veränderung des stationären Verformungs- oder Sickerwasserverhaltens hinweisen.

\section{Der Haselsteinspeicher}

Mit $40000 \mathrm{~m}^{3}$ Speicherinhalt dient das Becken zur Füllung des Speichers Oschenik, des größten Hochgebirgsspeichers der Kraftwerksgruppe Fragant, mit 33 Mio. m $^{3}$ Inhalt. Das ovale Becken ist von seiner Größe her der Topografie des Almplateaus angepasst und aus selektiertem Moränenund Bergsturzmaterial, vermischt mit Kieslagen, die aus der Möll antransportiert wurden, aufgebaut. Vorhandene Lehmschichten im Bereich des Beckens und der Dammfundierung mussten beseitigt werden und die angetroffenen Untergrundverhältnisse führten $\mathrm{zu}$ einer $1,5 \mathrm{~m}$ tieferen Gründung der Beckensohle. Den bodenmechanischen Erfordernissen entsprechend wurde auf das Erreichen eines Verformungsmoduls von $20 \mathrm{MPascal} / \mathrm{m}^{2}$ vor Einbau der weiteren Schüttlagen geachtet. Bei einer luftseitigen Dammneigung von 2:3 wurde noch ein klassisch aufgebauter Dammfuss aus Grobmaterial zur Sicherung und geordneten Drainagierung des Hanges angeordnet. Die Ausstattung des Speicherbeckens mit Hochwasserent-

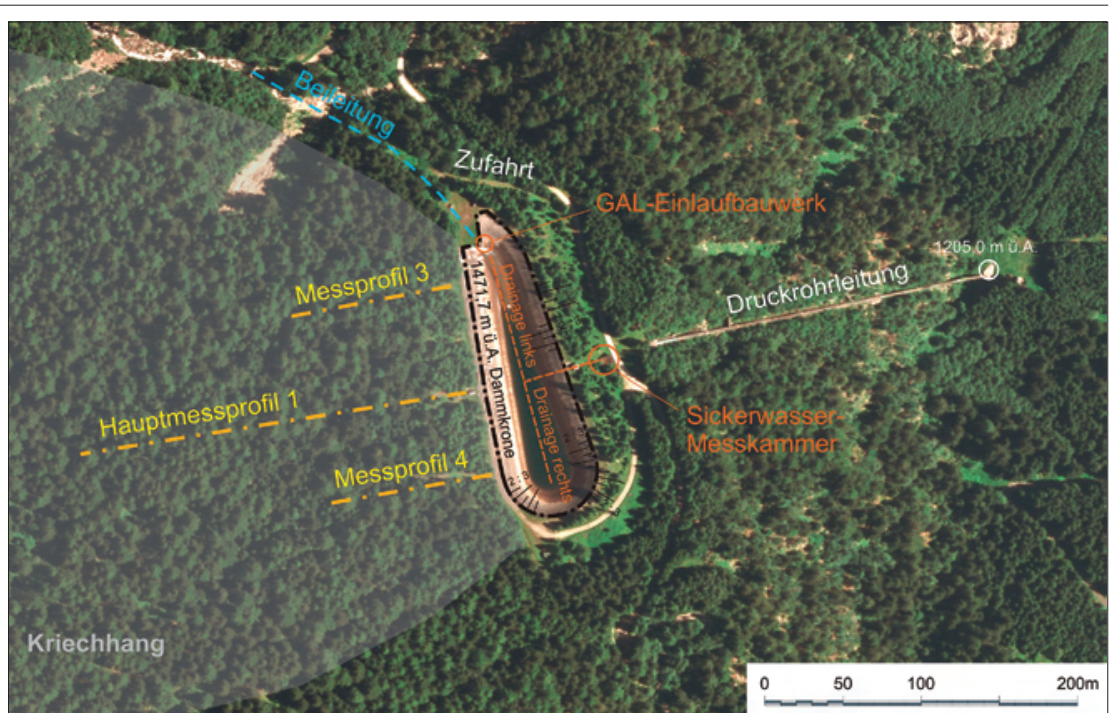

Abb. 2: Lageplan des Speicherbeckens.

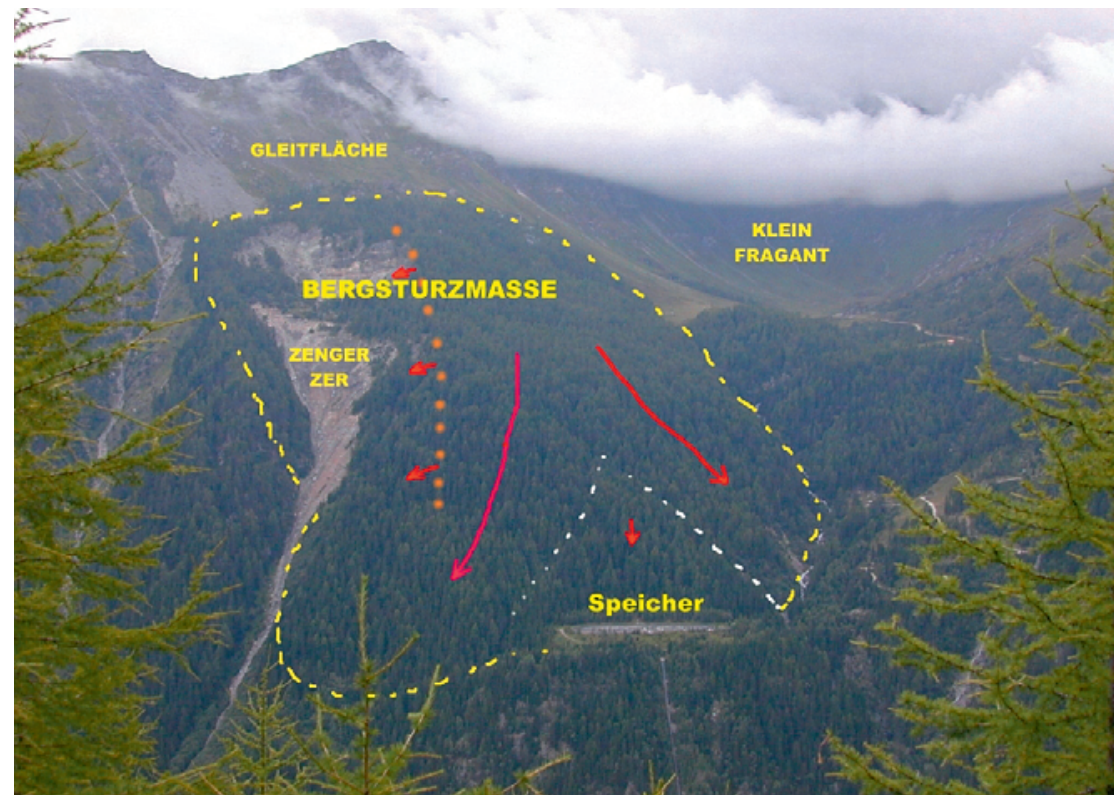

Abb. 3: Übersicht geologische Modellvorstellung.

lastung $\left(6,5 \mathrm{~m}^{3} / \mathrm{s}\right)$, Grundablass- und Restentleerleitung erfüllt noch die heutigen Anforderungen an derartige Sicherheitseinrichtungen.

\section{Der geologische „Background“}

Der Untergrund des Speichers Haselstein wird von unkonsolidierten Lockergesteinsmassen aufgebaut, die einer glazialen Terrasse aus anstehendem Gneis aufgelagert sind. Der Felsuntergrund weist, wie aus Aufschlussbohrungen bekannt ist, offene Klüfte auf, durch die eine Entwässerung der auflagernden Lockermassen erfolgt. Durch die 1996/97 ausgeführten Bohrungen wurde die Felsline in einer Seehöhe von 1456 bis $1459 m$ bis zu einer
Entfernung von $100 \mathrm{~m}$ bergseitig des Speicherrandes erbohrt. Es zeigt sich damit, dass die nahezu ebene Felsterrasse wesentlich größere Dimensionen hat als ursprünglich angenommen wurde.

Die der Terrasse auflagernden Sedimente bestehen zum größten Teil aus matrixgestützten Kies-, Stein- und Blockmassen. Die Matrix besteht aus Kies, Sand und Silt bei Überwiegen von Sand. Siltig-tonige Lagen treten nur selten und mit geringer Mächtigkeit auf. Gelegentlich sind Lagen mit Blockanreicherungen $\mathrm{zu}$ beobachten. Im mittleren Bereich wurde eine Brekzienlage mit geringer Ausdehnung und Mächtigkeit erbohrt.

Die Lockermassen wurden ursprünglich als Moränen gedeutet. Jüngere Unter- 


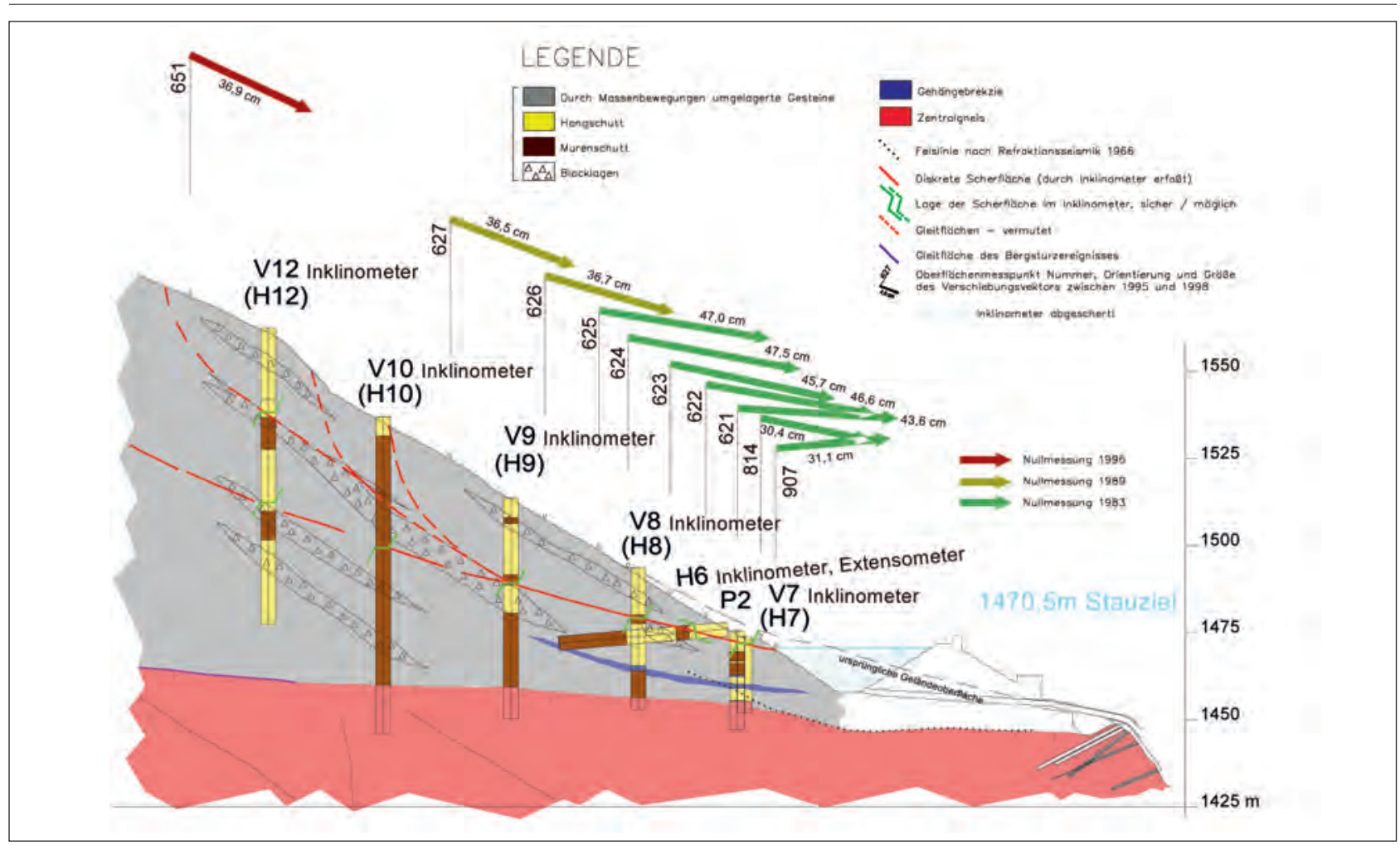

Abb. 4: Hauptmessquerschnitt mit Geologie und Verformung des Kriechhanges.

suchungen ergaben jedoch, dass es sich vorwiegend um Murenablagerungen bzw. durch Murenereignisse umgelagerte Ablagerungen einer riesigen postglazialen Massenbewegung handelt.

Die möglicherweise in Form eines einmaligen katastrophalen Ereignisses abgelaufene postglaziale primäre große Felsgleitung ist in ihrem Stirnbereich auf die flache breite Felsterrasse aufgefahren und dort zum Stillstand gekommen. Durch die hohe Durchlässigkeit des Felsuntergrundes und die damit verbundene Entwässerung der Lockermassen hat sich der zentrale Fußbereich im Laufe der Zeit konsolidiert und ein Widerlager gebildet.

Durch die andauernde Erosion im Zenger Zer-Gebiet wird der südliche Teil der Gleitmasse weiter destabilisiert und gibt ständig erhebliche Materialmengen in die Zenger Zer ab. Dadurch kommt es zu Kippbewegungen der aufgelockerten Felsschollen der Gleitmasse gegen SO, wie in Abbildung 3 dargestellt ist.

In ähnlicher Weise, wenn auch in wesentlich geringerem Ausmaß, führt die Erosionsleistung des Kleinfraganter Baches am Nordrand der Gleitmasse zur Entlastung des Böschungsfußes und damit zu einem Nachdrängen der Lockermassen.

Durch diese andauernde Erosion im Randbereich der alten Gleitmasse wird diese destabilisiert und es kommt zu se- kundären Massenbewegungen innerhalb der alten Ablagerungen.

Diese bis heute andauernden sekundären Materialverlagerungen erfolgen in Form von

- Blockstürzen

- Kippen von Felsschollen

- diffusem Kriechen

- oberflächennahem Schuttfließen und Schuttkriechen

- Gleiten auf diskreter Gleitfläche

Stürzen und Kippen bleiben auf die seitlichen Erosionsbereiche beschränkt. Kriechen tritt im gesamten Bereich auf.

Gleiten ist am Hangfuß bergseits des Speichers zu beobachten und letztlich für die Ausbildung des Wulstes an der Dichthaut verantwortlich.

Die andauernden aktiven Bewegungen des Hanges sind seit 1983 durch mindestens eine jährliche Messung dokumentiert, wie in Abbildung 4 dargestellt.

\section{Die Betriebserfahrungen}

Über die ersten 12 Betriebsjahre finden sich in den Archiven der KELAG keine Aufzeichnungen. Dies erklärt sich wohl aus dem Umstand, dass in den 70er Jahren die Kraftwerksgruppe Fragant baulich entscheidend erweitert wurde und zu wenig Personalressourcen wie auch fehlendes
Gefahrenbewusstsein für eine regelmäßige Sperrenüberwachung bestand. Die gravierenden Probleme der Kölnbreinsperre bei den ersten Vollstauversuchen Ende der 70er Jahre haben auf behördlichen Druck zu entscheidenden Veränderungen bei der Talsperrenüberwachung geführt. So wurde bei allen Dämmen der KELAG an den Sickerwassersummenmessstellen ein Messwehr eingebaut und mit Grenzwertschaltbirnen ausgestattet.

Hinsichtlich der Langzeitfunktion sind die Erfahrungen mit Asphaltdichtungen in Speicherbecken europaweit sehr gut. Beim Haselsteinspeicher wurde 1979 erstmalig eine wulstartige Verformung der bergseitigen Böschung festgestellt, die sofort örtlich saniert wurde. Ab diesem Zeitpunkt sind geodätische Überwachungskampagnen überlegt worden, die auch zu einem Einbau einer größeren Anzahl von Oberflächenpunkten führte, die mindestens einmal jährlich vermessen wurden. Es stellte sich bald heraus, dass der talseitige Damm keine Verformung erleidet, jedoch am bergseitigen Hang ein kontinuierliches Kriechen einer oberflächlichen Schichte die Asphaltfläche massiv in Mitleidenschaft zieht. Neben der Verformungsproblematik gibt es beim Speicherbecken Haselstein auch eine Pustelentwicklung, für die die Fachwelt des Asphaltwasserbaus noch keine genauere Ursachenerklärung 


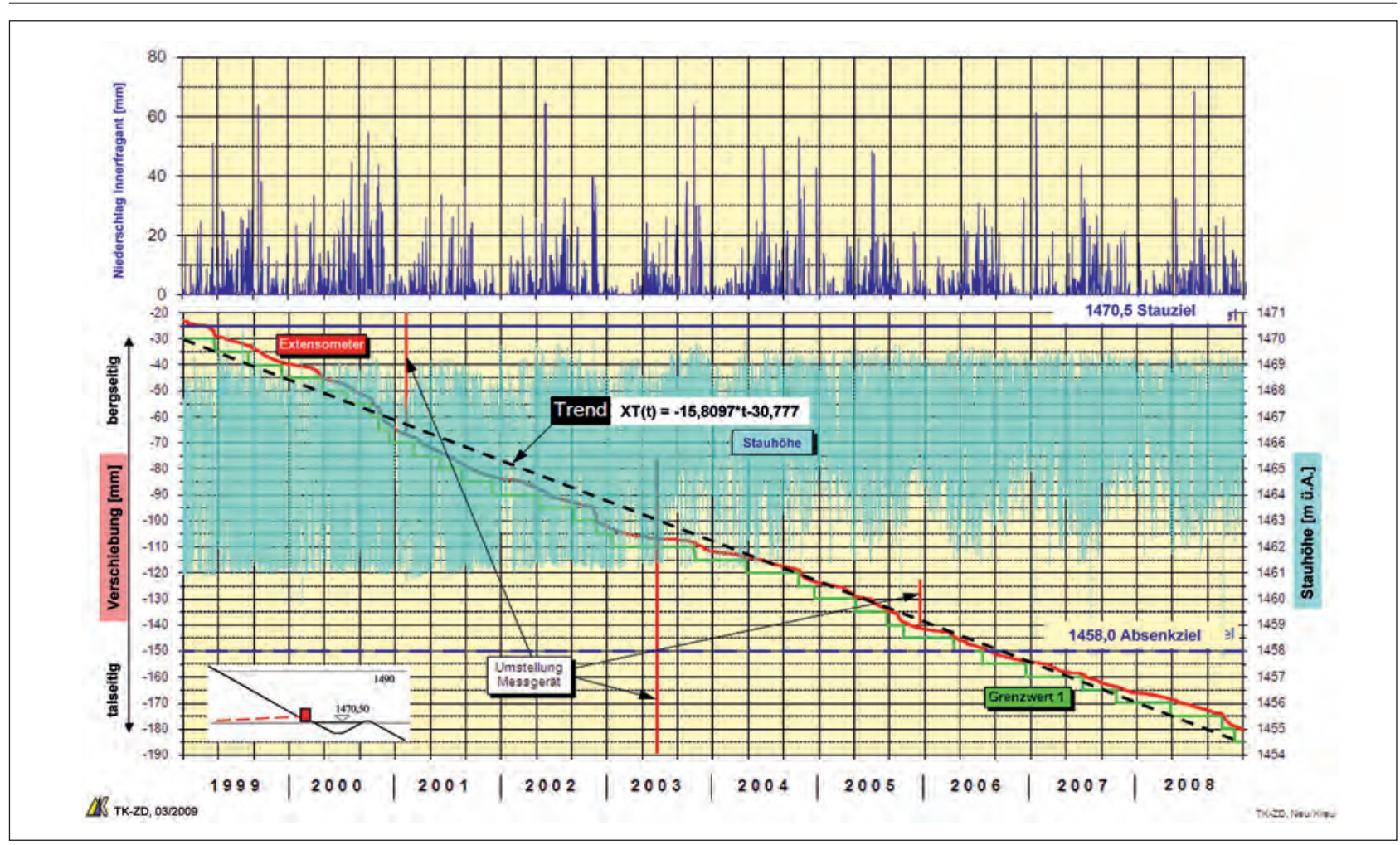

Abb. 5: Niederschlag und Extensometermesswertaufzeichnung 1999-2008.

gefunden hat. Nussgroße Hohlraumbildungen in der oberen Dichtschichte des Asphaltes verleihen der Oberfläche eine warzenähnliche Struktur und mit jedem Jahr nehmen Pustelanzahl und Größe zu. Nach anfänglich örtlichen Sanierungen und Überklebungen von ausgeprägten Pustelflächen ist nunmehr eine Generalsanierung in Form von klassischem Abfräsen der bestehenden Dichtungsschichte und Neueinbau einer Dichtlage von $8 \mathrm{~cm}$ vorgesehen.

\section{Die Mess- und Beobachtungs- einrichtungen in ihrer zeitlichen Entwicklung}

Abhängig von den Anforderungen an eine zeitgemäße Talsperrenüberwachung wurden die Mess- und Überwachungseinrichtungen in den letzten 30 Betriebsjahren laufend erweitert:

- 1968 - Inbetriebnahme, Drainageausleitung zur Sickerwassermessstelle (manuelle Messung); Pegel- und Zuflussmesswertübertragung ins KH Innerfragant

- 1981 - Einbau eines Messwehrs bei der Sickerwassersummenmessstelle mit Grenzwertgeber und Übertragung der Werte in die ständig besetzte Hauptschaltleitung (Einführung des geregelten Bereitschaftsdienstes für Talsperrenverantwortliche)
- 1983 - Einbau von ersten geodätischen Oberflächenmesspunkten an der Dammkrone, Asphaltböschung sowie bei zwei bergseitigen Hangprofilen zur Verformungsdokumentation

- 1990 - Einbau der ersten zwei Inklinometerrohre in geologische Aufschlussbohrlöcher am bergseitigen Beckenrand zur Erfassung der Untergrundverformung

- 1991 - Online-Sickerwassermesswertübertragung und regelmäßige Protokollierung sowie Installation zweier zusätzlicher Grenzwertgeber

- 1994 - Erweiterung der geodätischen Messprofile und Einbau von Oberflächenpunkten an der Schulter des Kriechhanges (bis $600 \mathrm{~m}$ über dem Speicherbecken auf $2000 \mathrm{~m}$ über Adria)

- 1995 - Einbau eines 42 m langen Stangenextensometers im Hauptmessquerschnitt im Bereich des Beckenrandes zur Online-Überwachung der Hangkriechbewegung, eines horizontalen Inklinometerrohres zur Verformungsmessung des Untergrundes, sowie Einbau dreier Piezometerrohre im Gelände zur Bergwasserbeobachtung

- 1996 - Geologische Geländeaufnahme des Kriechhanges durch MitarbeiterInnen der TU Graz und Ausweitung der geologischen Aufschlussbohrkampagnen
- 1997 - Einbau von weiteren Inklinometerrohren in geologischen Aufschlussbohrlöchern zur Verformungsmessung des Untergrundes im Hauptmessquerschnitt (Aufschliessungsarbeiten waren für die Einreichung zur Endkollaudierung 1998 von Bedeutung)

- 2004 - Umstellung der Datenübertragung von Analog- auf Digitaltechnik

- 2009 - Einrichten einer Videokamera im Speicherbereich sowie redundante Pegelmesseinrichtung für den Wasserstand im Becken

\section{Auswertung der Verformungsmessungen}

Das Verständnis für die Massenbewegung konnte durch die geologischen Aufschlussbohrungen und vor allem durch die regelmäßigen Messungen von über 90 Oberflächenpunkten hinsichtlich der Kinematik des Hanges wesentlich gesteigert werden. Zur Untermauerung der geologischen Modellvorstellung wurde das Messnetz über den Kriechhang immer mehr ausgedehnt. Überlegungen einer GPS-Vermessung scheiterten leider im Jahr 2000 an der ungünstigen Hangausrichtung gegen Osten, wo die Verfügbarkeit von ausreichend vielen Satelliten nicht einwandfrei gegeben war.

Im oberen Teil des Hauptmessprofiles haben sich die größten Beträge von $35 \mathrm{~cm}$ 


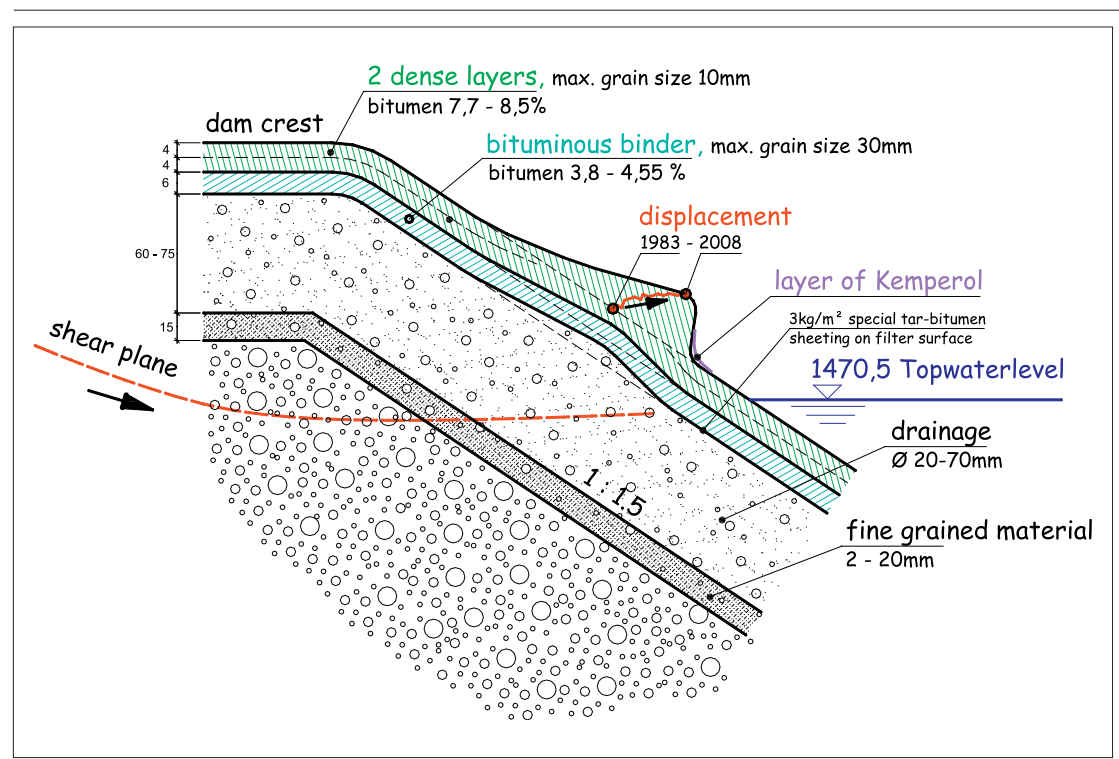

Abb. 6: Verformung der Asphaltoberfläche durch Hangkriechen 1980-2008.

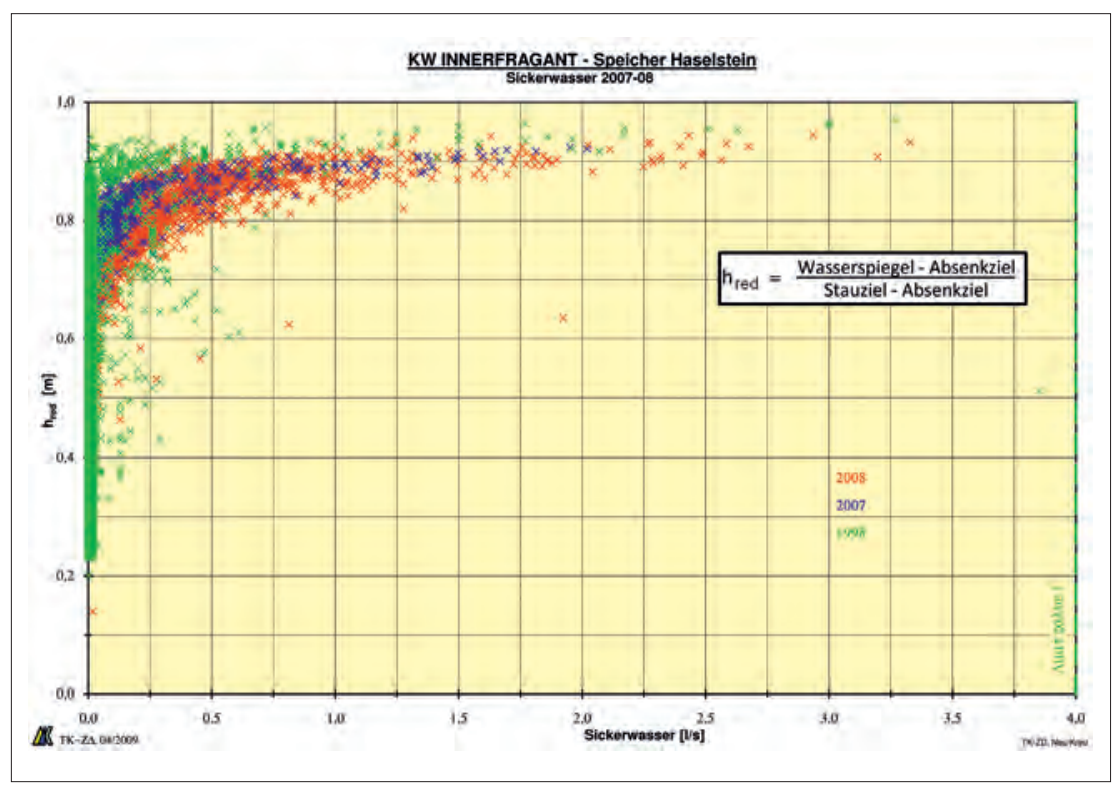

Abb. 7: Stauabhängiges Sickerwasserverhalten 1999, 2007, 2008.

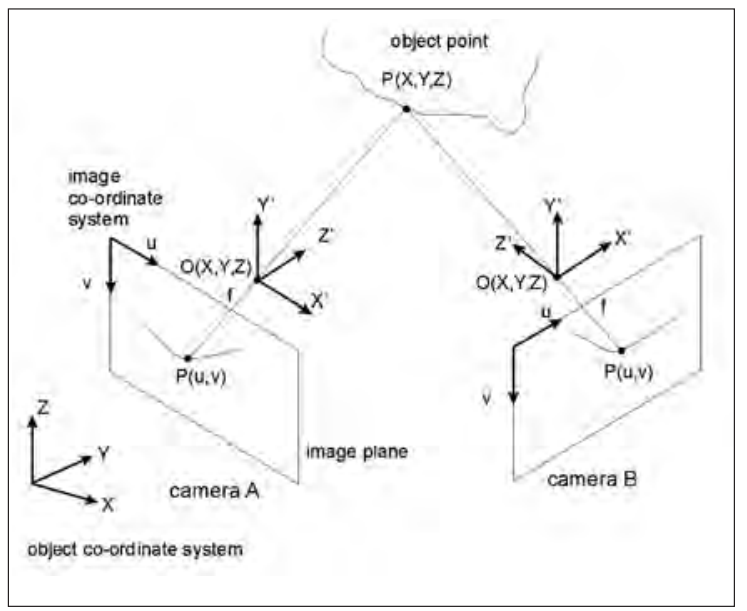

Abb. 8: Prinzip der raümlichen Fotografie.
Verschiebung sowie $25 \mathrm{~cm}$ Setzung in einem Zeitraum von 13 Jahren ergeben. Auch die Punkte in der Nähe des OnlineExtensometers beim Beckenrand verifizieren das konstante Kriechverhalten des Hanges, das in diesem Bereich nur mehr halb so groß ist. Durch die kontinuierliche Aufzeichnung der Extensometerverformung ist ein deutlicher Zusammenhang zwischen der Verformungsgeschwindigkeit und Niederschlägen sowie Schneeschmelzperioden festzustellen. Während der letzen 10 Jahre ist es zu keinem exponentiellen Anstieg der Verformung gekommen. Die Trendanalyse der Messdaten ergibt den linearen Zusammenhang von

$X T(t)=-15,8097 t-30,777, X(t)$

in mm-Einheit, wie in Abbildung 5 dargestellt.

Diese Kriechbewegung bewirkt im Speicherbereich eine Wulstbildung, die in der Zwischenzeit schon nahezu $40 \mathrm{~cm}$ erreicht. Durch den ständigen, aktiven Erddruck versucht sich die Asphaltdecke der Lastaufnahme durch die Wulstbildung zu entziehen. Wegen dem flachen Felsplateau im Untergrund, der Auflast der Überlagerung und der Tatsache, dass der Hang beim Bau des Beckens übersteilt wurde, wird im Bereich des Beckenrandes das Material nahezu horizontal verdrängt. Geodätisch erfasste Oberflächenpunkte, die auf der Asphaltdichtung fixiert sind, zeigen eine horizontale Verformung von beinahe $40 \mathrm{~cm}$ bzw. eine Hebung von einigen Zentimetern, wie in der Prinzipskizze Abbildung 6 dargestellt. Dies ist eindeutig auf die gute Bitumenqualität (hohe Penetration) zurückzuführen, da es auch nach 40 Jahren noch zu keiner großen Versprödung des Materials gekommen ist.

Die zähplastischen Eigenschaften des Asphaltmaterials ermöglichen Verformungen, die noch zu keinen Rissbildungen geführt haben. Die derzeitige Situation drängt nach einer Sanierung, die noch 2009 vorgenommen werden soll. Durch die teilweise flächenhafte Verschiebung des bergseitigen Beckenbereiches und zum Studium der Alterung der Asphaltoberfläche wurde dieser Bereich bereits 2004 mit einer Spezialkamera abgebildet, wie im nächsten Kapitel beschrieben ist.

Das entscheidende Kriterium für die Sicherheit des Staubeckens ist die Dichtheit. Hier zeigte sich im Laufe der letzten drei Jahrzehnte ein Sickerwasserandrang nur in den oberen zwei Metern des Staubereichs. Ursache könnten sich öffnende Bahnnähte 


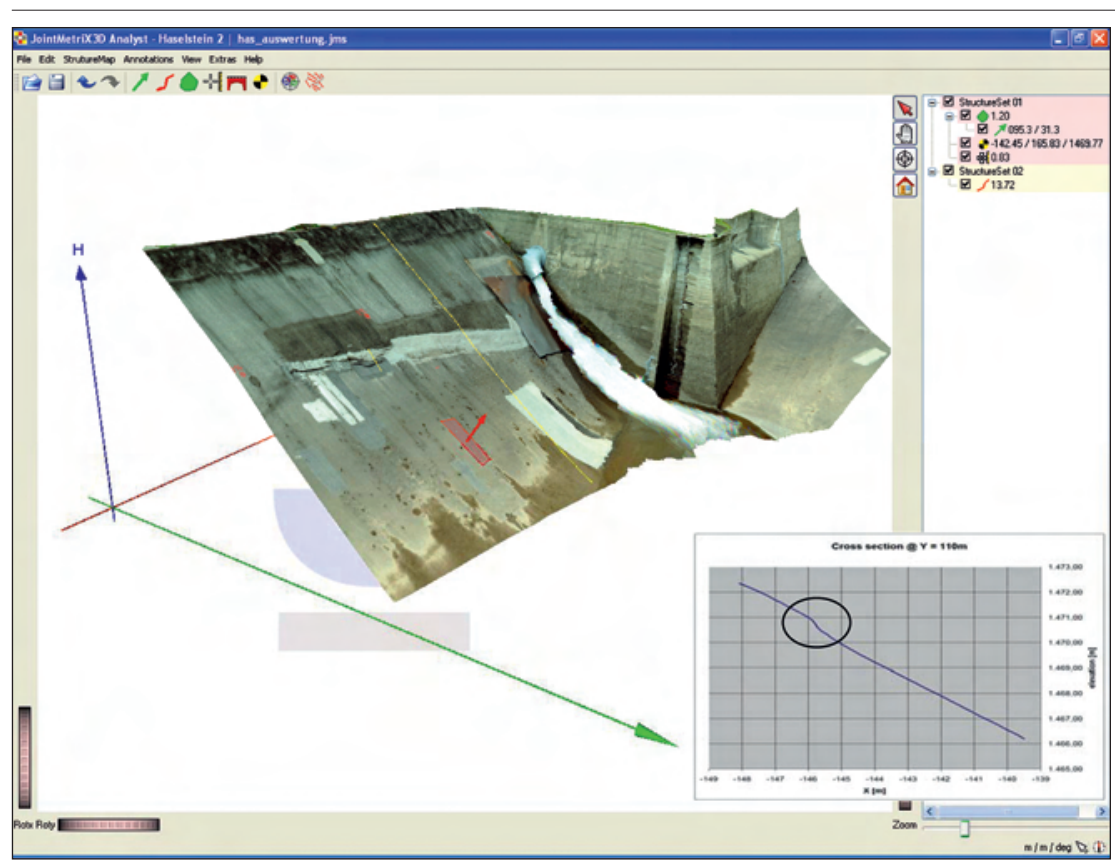

Abb. 9: Bildschirmfoto der 3D Aufnahme des Haselsteineinlaufbereiches.

der Asphaltdichtung sein, obwohl die zweilagigen Bahnen versetzt ausgeführt worden sind. Der obere Bereich des Beckens ist auch empfindlicher auf allfällige Setzungen. Trotz umfangreicher Färbeversuche gelang es nicht die Eintrittsstellen zu orten. Mit punktuellen Sanierungen mittels Spezialbandagen konnte bisher die Sickerwassersituation stationär gehalten werden, wie in Abbildung 7 gezeigt wird.

\section{Dokumentation der Oberfläche mit JointMetrix3D}

JointMetriX3D ist ein System zur Erfassung von Gebirgs- und Geländeoberflächen mittels dreidimensionaler Bilder. Es nutzt Prinzipien der Photogrammetrie und der Computer Vision. Bestandteil des Systems ist eine hochauflösende Panoramakamera, die sehr große Bilder mit 100 Megapixel und sogar darüber hinaus erzeugen kann. Die Anzahl der Pixel ist entscheidend für die Anwendung in der Objektdokumentation, da diese direkt die Auflösung an der Objektoberfläche beeinflusst: je höher die Auflösung, desto besser die Möglichkeiten für die späteren Bewertungen.

Aus einem Bildpaar wird mit modernen Algorithmen ein dreidimensionales Bild automatisch errechnet, wobei das 3D Bild die Vereinigung der fotografischen und geometrischen Informationen darstellt. Abbildung 8 zeigt schematisch wie aus zwei Bildern eine räumliche Rekonstruktion durchgeführt werden kann.

Ein typisches 3D-Bild der Asphaltober- fläche besteht aus mehreren hunderttausend Oberflächenmesspunkten. Die Bildauflösung beträgt im konkreten Fall etwa $3 \mathrm{~mm}$. 3D-Bilder stellen daher eine sehr gute Datengrundlage für die Erfassung des aktuellen Zustands der Asphaltoberfläche dar. Sie sind eine objektive, reproduzierbare Dokumentation, aus denen zusätzlich geometrische Messungen gewonnen werden können.

Durch wiederholte Aufnahmen ist es möglich, Bewegungen über die Zeit aufzuzeichnen und zu quantifizieren, z.B. über den Vergleich von Profilschnitten, Verschiebungsvektoren oder Volumsänderungen.

Neben der permanenten Dokumentation der Oberfläche wird auch die Planung da z. B. das Ausmaß beschädigter Oberflächenanteile gemessen werden kann, bevor diese saniert werden.

Es ist wichtig den Mechanismus und die Kinematik der Bewegungen zu erkennen. Dies kann nur über Langzeitbeobachtungen erfolgen. Eine Erweiterung des verwendeten 3D-Bilderfassungssystems gestattet nun die Ergänzung einzelner Bereiche durch Aufnahmen, die mit einer handelsüblichen, aber kalibrierten Digitalkamera erzeugt werden.

Die bestehenden hochauflösenden 3D-Bilder dienen als Referenz für die zusätzlichen Aufnahmen mit dem Fotoapparat, d.h. keine weiteren äußeren Bezugspunkte sind notwendig. Die Fotos können freihändig ohne Bestimmung der Aufnahmestandpunkte erzeugt werden. Eine von Sanierungsmaßnahmen unterstützt, durchgehende Datenerfassung und Überwachung wird damit auf zwei Arten unterstützt: (i) durch häufigere, weil einfachere 3D-Bilderfassung speziell in problematischen Bereichen, wobei vor Ort einfach Fotos gemacht werden müssen und (ii) wenn zu einem Aufnahmezeitpunkt Teile der Asphaltoberfläche nicht sichtbar waren (z. B. durch einen zu hohen Wasserpegel oder eine Schneedecke) werden diese einfach zu einem späteren Zeitpunkt ergänzt. Für den Speicher Haselstein ist ein Ausschnitt in Abbildung 9 dargestellt.

Das System der wasserseitigen Asphaltdokumentation mittels Spezialkamera wird auch bei anderen Dämmen mit Asphaltoberflächendichtung in hochalpinen Gebieten eingesetzt.

\section{Zusammenfassung}

Die außergewöhnlichen Verformungserscheinungen der Oberfläche, die sich im Becken das erste Mal 1979 zeigten, haben umfangreiche Überwachungs- und Untersuchungskampagnen ausgelöst, um mehr Verständnis für ein mögliches Versagenszenario zu gewinnen. Redundante Messeinrichtungen sichern aufgrund der Messergebnisse die Beurteilungen $\mathrm{ab}$ und bestärken den/die verantwortliche/n IngenieurIn in seiner/ihrer Verantwortung für die Sicherheitsbeurteilung der Anlage, sodass es auch zu vertreten war, die überfällige Asphaltsanierung einige Jahre hinauszuschieben.

\author{
Korrespondenz: \\ F. Neuschitzer \\ KELAG - Kärntner Elektrizitäts-Aktiengesellschaft \\ Arnulfplatz 2, A-9020 Klagenfurt \\ K. Klima \\ Technische Universität \\ Rechbauerstraße 12, A-8010 Graz \\ A. Gaich \\ 3G Software \& Measurement GmbH \\ Plueddemanngasse 77, A-8010 Graz
}

\section{LITERATUR}

Nackler K, Neuschitzer F, Riedmueller G, Klima K (2000) „Speicher Haselstein - Risikoabschätzung durch spezielle Überwachung“, Schriftenreihe zur Wasserwirtschaft, Techn. Univ. Graz, Band 34 „Betrieb und Überwachung wasserbaulicher Anlagen"

Neuschitzer F, Klima K, Gaich A (2005) "Recording and surveillance of ageing asphaltic concrete linings by metric 3D imaging", Proc. of the Int. Hydro (2005)Conference, Villach, Austria, Waterpor ria, Waterpower and Dam Construction, pp.1-8 Berger G, Neuschitzer F (2003) "Mining the Data "Journal of International Water power an Dam Construction, London, p.38-42 